\title{
Achieving Open-loop Insulin Delivery using ITM Designed for T1DM Patients
}

\author{
Akash Rajak \\ Associate Professor, Krishna Institute of Engineering \& Technology, Ghaziabad (U.P), India \\ Email: akashrajak@rediffmail.com
}

Kanak Saxena

Professor, Samrat Ashok Technological Institute, Vidisha (M.P), India

Email: ksv1909@yahoo.com

\begin{abstract}
To simulate the glucose-insulin concentration of type 1 diabetic patient an Intelligent Temporal Mediator (ITM) has been designed. The ITM integrates the tasks of temporal reasoning and temporal maintenance. The paper discusses the design of ITM reasoning system which was based on open-loop insulin delivery technique. The result shows that ITM successfully models the blood glucose profile of the diabetic patient. The designed ITM is also compared with existing open-loop simulator for checking its performance.
\end{abstract}

Index Terms-temporal mediator, temporal reasoning, temporal maintenance, T1DM.

\section{Introduction}

Almost all medical data are time stamped or timeoriented (e.g. patient measurements, therapy interventions), so it is virtually impossible to plan therapy, apply the therapy plan, monitor its execution, and assess the quality of the application or its results without the concept of time [1]. Temporal data mining is concerned with analysis of ordered datasets with temporal interdependencies [2, 3]. The research in the field of temporal data mining has two directions. One is temporal maintenance and another is temporal reasoning. The task of temporal maintenance is investigated by researchers of database community, often associated with management of time-oriented databases. Temporal reasoning is related to intelligent analysis of time-oriented databases and is related to artificial intelligence. Shahar et al. [4] integrated the task of temporal reasoning and temporal maintenance through a mediator known as temporal mediator. The temporal mediator forms a distinct middle layer making the user application independent of data resources [5, 6]. The temporal mediator is highly desirable in the field of medical research $[7,8]$.

The remainder of this paper is organized as follows: Section 2 gives an overview of Intelligent Temporal Mediator (ITM). Section 3 describes how the design of ITM utilizes the concept of open-loop insulin delivery. Section 4 illustrates the temporal reasoning tasks performed by ITM. Section 5 presents the implementation of Intelligent Temporal Mediator. The Section 6 gives the testing of the ITM. Conclusion and future directions work are given in the final section.

\section{Intelligent Temporal Mediator}

We designed a temporal mediator called as Intelligent Temporal Mediator (ITM) to forecasts the blood glucose concentration of patient having type 1 diabetes mellitus (T1DM). In T1DM the patient has to depend on external insulin for regulating the blood glucose levels. The exogenous insulin can be infused in the patient's body by 
either closed-loop or open-loop insulin delivery techniques.

The closed-loop insulin infusion is an automated process controlled by computer algorithm. The closedloop insulin delivery system acts as artificial pancreas, which senses plasma insulin concentration in the blood, calculates the amount of insulin needed by the body for maintaining normoglycemia level and finally delivers the correct amount of insulin [7]. The system is composed of glucose sensor, an insulin pump and control algorithm for regulating the insulin pump. Open loop methods of insulin delivery focus on a patient administering insulin to his or herself at different times of the day [7]. It is based on taking multiple injections using a combination of short and long acting insulin analogues supported by blood glucose self monitoring [7]. Normally the patient has to take 3-4 injections daily for regulating the plasma glucose level in the blood. The physician suggests a diet chat for the patient and prescribes him the quantity and type of injections he has to take at various time intervals for maintaining normoglycemia levels.

The ITM integrates the tasks of temporal reasoning and temporal maintenance using ITM reasoning system and ITM maintenance system respectively. The ITM reasoning system performs the task of temporal abstraction for creating interpretations of time-stamped data in terms of higher-level concepts and patterns that hold over time intervals. The system does visualization and exploration of patient's blood glucose concentration in form of the smooth curves plotted over various bands range. The temporal patterns are based on value of blood glucose concentrations on some time-intervals. The temporal pattern accounts for hypo and hyper blood glucose episodes.

The ITM maintenance system stores temporal data of diabetic patient. The temporal data is related to patient's history, laboratory reports, physical examinations, therapy schedule etc.

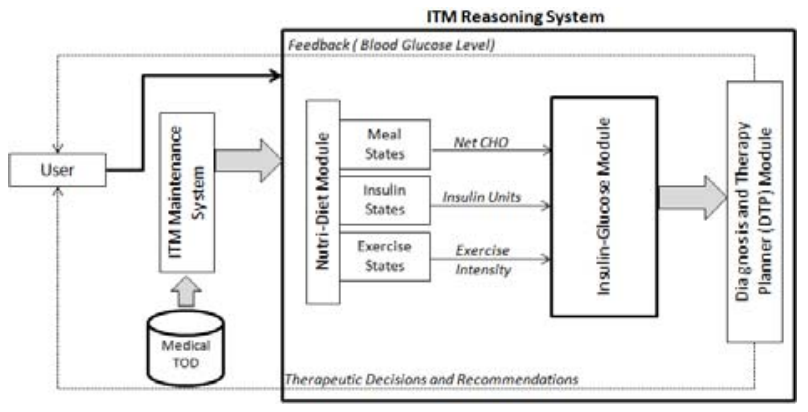

Fig 1: The ITM consists of reasoning and maintenance systems. The ITM reasoning system accepts inputs from User or ITM maintenance system. To forecast blood glucose concentration of T1DM patient ITM reasoning system uses Bergman minimal model [9, 10,11, 12, 13, 14, 15], Fisher model [16] and Roy exercise model [17].

\section{Achieving Open-loop Insulin Delivery}

The design of ITM reasoning system was based on open-loop insulin delivery $[7,8]$. The ITM Reasoning system consists of three modules: Nutri-Diet module, Insulin-Glucose module, and Diagnosis and Therapy Planner module. To achieve open-loop insulin delivery the Nutri-Diet module defines six, four and two states for meal intake, exogenous insulin infusion and physical exercise respectively.

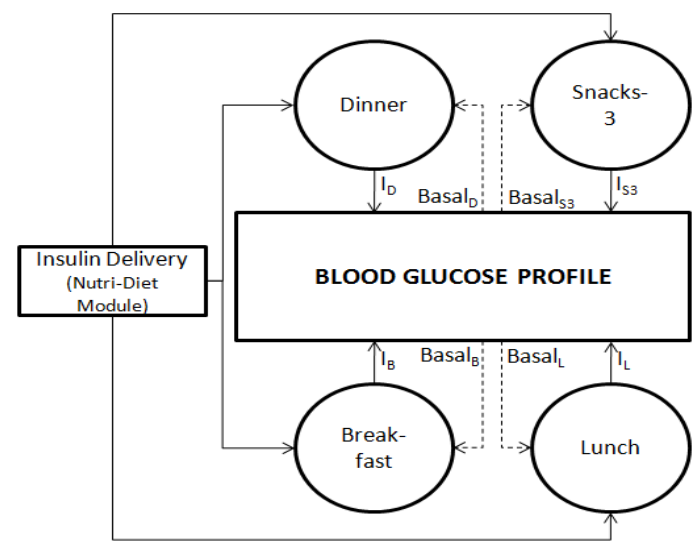

Fig 2: Four states for insulin delivery are defined for achieving openloop insulin delivery. The insulin can be induced during any of these states, externally. $I$ denote the units of insulin induced in patient's body and Basal denotes the base value of the insulin.

From the Figure 2, the blood glucose profile of the diabetic patient accepts exogenous insulin infusion during four states. The exogenous insulin absorption during these four states reduces the blood glucose concentration of the patient. The external absorption of glucose in form of carbohydrates $(\mathrm{CHO})$ can be achieved by six meal states. 
These six meal states acts as disturbance in the blood glucose profile of the patient as it increases the blood glucose level of the patient. The two states are defined for physical exercises. The physical exercise performed by the patient reduces the blood glucose concentration.

\section{Temporal Reasoning}

The temporal reasoning (TR) tasks performed by ITM reasoning system are planning, data extraction, projection, forecasting and diagnosis.

\subsection{TR Tasks of Nutri-Diet Module}

The Nutri-Diet module will be responsible for planning, data extraction and projection.

The task of planning consists of producing a sequence of actions for a care provider, given an initial state of the patient and a goal state, or set of states, such that sequence achieves one of the patient goal patient states [4]. The task of planning defines various states of NutriDiet module.

The module performs the tasks of data extraction on mixed meal and exercise data. All these data are collected on various time-stamps. From the mixed meal the module extracts the contents of carbohydrates, fats, saturated fats and cholesterol. The module extracts the intensity of exercise from the type of exercise performed by the patient.

The projection is the task of computing the likely consequences of set of conditions or actions, usually given as a set of cause-effect relations [4]. For e.g. fifteen grams of carbohydrate should raise your blood glucose about $30 \frac{\mathrm{mg}}{\mathrm{dL}}$ to $40 \frac{\mathrm{mg}}{\mathrm{dL}}$ in 10 to 15 minutes. The Nutri-Diet module projects the value of net $\mathrm{CHO}$ intake based on GI (glycemic index) and GL (glycemic load) of the carbohydrates. The net $\mathrm{CHO}$ means the quantity of $\mathrm{CHO}$ that affects the glycemic level of blood sugar.

\subsection{TR Tasks of Insulin-Glucose Module}

The Insulin-Glucose module performs the tasks of forecasting. It involves predicting particular future values for various parameters given a vector of time-stamped past and present measured values [4]. The module forecasts the blood glucose concentration and the effect of carbohydrates (CHO) on blood glucose profile of a patient having T1DM.

\subsection{TR Tasks of DTP Module}

The DTP module performs the tasks of diagnosis and therapy planning. The Insulin-Glucose module projects the blood glucose concentration of the patient by utilizing various models of insulin-glucose metabolism [7] and abstracts the information in form of temporal patterns. The temporal patterns are evaluated by DTP module for performing the task of diagnosis. Each temporal pattern may fall either in three bands range. These temporal patterns are evaluated in terms of various blood glucose episodes by the DTP module. After successful diagnosis the module plans for a therapy.

\section{ITM Implementation}

The ITM reasoning system adopts various models of glucose-insulin metabolism to forecast the blood glucose (BG) concentration of the diabetic patient. The Bergman minimal model [15] was utilized for studying the BG concentration of diabetic patient. The minimal model is integrated with Fisher model [16] for producing meal disturbance from mixed meal. One parameter of the Fisher model is modified to create disturbance obtained from net $\mathrm{CHO}$ intake during six states. Presently the design of ITM considers only the effect of CHO on BG concentration. Further the exercise model is incorporated in the existing design of Insulin-Glucose module for tighter glucose control and is defined as two states. The physical exercise model was adapted from Roy et al. [17]. 


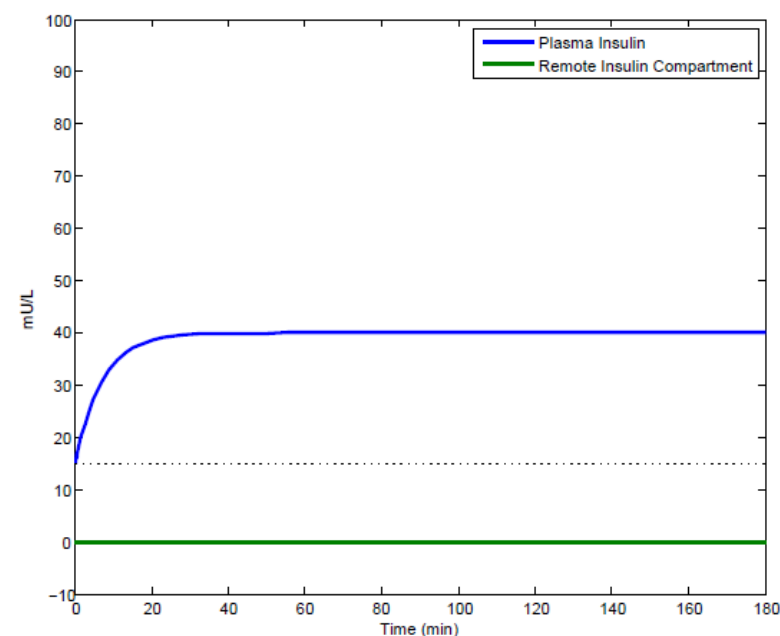

Fig 3: Plasma insulin concentration and insulin concentration in remote compartment. The black dotted line denotes the basal value of plasma insulin, which is assumed to be $15 \frac{\mathrm{mU}}{\mathrm{L}}$.

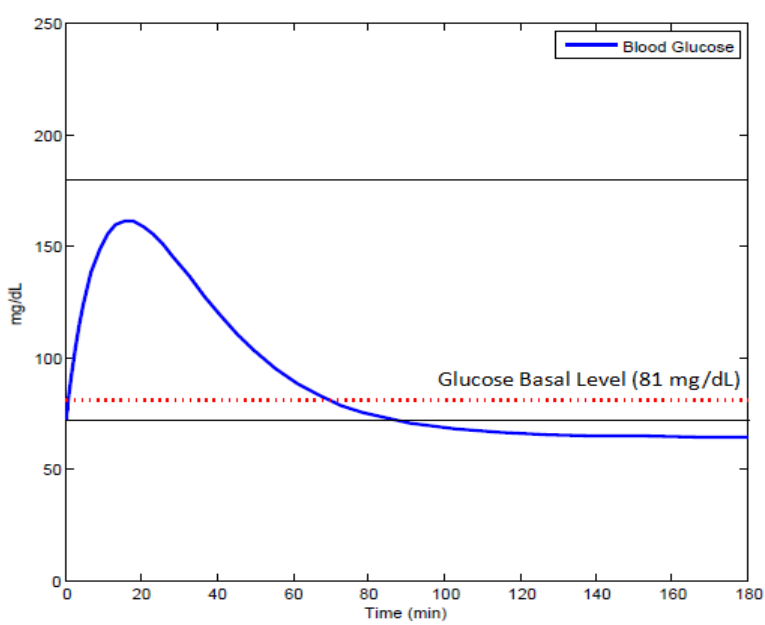

Fig 4: The red dotted line denotes the basal or fasting blood glucose level, normally recorded before Breakfast or 180mins after any meal.

The Bergman minimal model has three compartments consisting of plasma insulin, remote insulin, and blood glucose. The remote insulin compartment actively takes part in promoting uptake of plasma glucose into the liver and peripheral (extra-hepatic) tissues. To predict the behavior of Bergman minimal model, we implemented the model in MATLAB. The output of the Bergman minimal model is shown via Figures 3 to 4.

In Figure 3, shows the statistics of plasma insulin and insulin in remote compartment. The insulin level rises from basal value $15 \frac{\mathrm{mU}}{\mathrm{L}}$ and will remain constant to $30 \frac{\mathrm{mU}}{\mathrm{L}}$ after 106 mins. As there is no excess insulin infusion, the flow of insulin from remote compartment is $0 \frac{m U}{L}$. The Figure 4 represents the basal level of blood glucose concentration. The basal level is the normal level of blood glucose at $81 \frac{m g}{d L}$ in healthy patient.

\subsection{Bands Range}

A time series based methodology is used for representing the blood glucose values in the form of band ranges. Three band ranges have been define: (i) safety band, (ii) lower band, and (iii) upper band. The safety band corresponds to normoglycemia range between 72$180 \frac{\mathrm{mg}}{\mathrm{dL}}$. If the blood glucose level is below $72 \frac{\mathrm{mg}}{\mathrm{dL}}$ it will fall in lower band, while if it exceeds the value $180 \frac{\mathrm{mg}}{\mathrm{dL}}$ it will fall in upper band. The blood glucose values falling under lower or upper represents the unconscious state of patient, while in safety band represents the normoglycemia range [18].

\subsection{Temporal Patterns}

The Insulin-Glucose module forecasts the blood glucose concentration of the diabetic patients. The module abstracts in form of temporal patterns defined over various meal states. The temporal pattern over a meal state consists of BG values over a time interval. These temporal patterns are evaluated by Diagnosis and Therapy Planner (DTP) module in search of blood glucose episodes. 


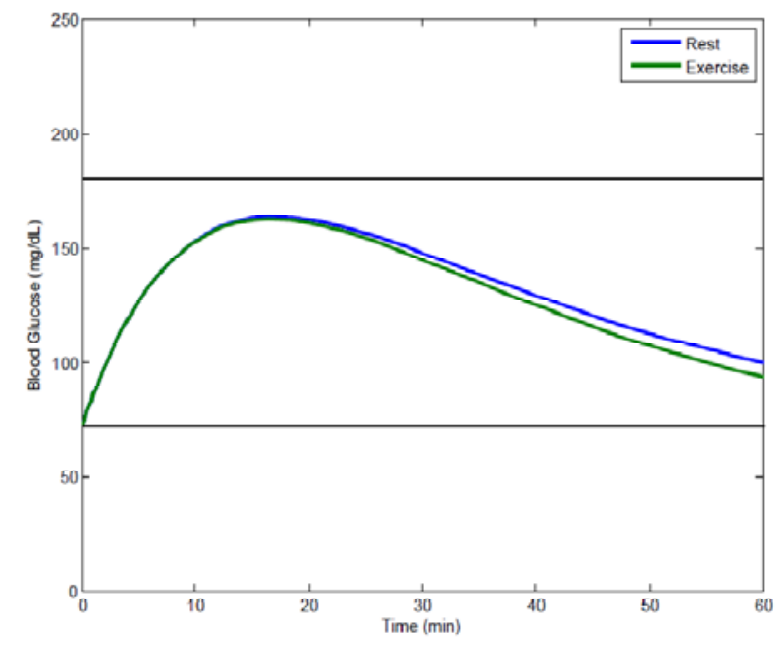

Fig 5: Variation in blood glucose level during rest and exercise of a diabetic patient, modeled by integrating Roy et al. [17] exercise model with the Bergman minimal model [15]. The result shows that adopting a physical exercise significantly lowers the blood glucose level and hence reduces the risk of hyperglycemia state.

The interval based temporal abstraction obtained by Insulin-Glucose module is passed to the Diagnosis and Therapy Planner (DTP) module for performing temporal reasoning related to patient's blood glucose level. The temporal patterns are defined in terms of blood glucose episodes by DTP module. The various blood glucose episodes defined are hypo and hyper episodes. The hyper episode represents severely high blood glucose value $\left(>180 \frac{m g}{d L}\right.$ ), while hypo episode represents extremely low blood glucose value $\left(<70 \frac{m g}{d L}\right)$.

\section{Testing}

The designed Intelligent Temporal Mediator is then tested for its performance and accuracy. The testing was based on various test cases adopted from the case history of AIDA simulator [19]. The ITM was tested on blood glucose episodes occurred due to hypoglycemia or hyperglycemia states. The result shows that the ITM successfully reasons the profile of T1DM patient. One of the test cases is discussed below.

The case is as follows: The patient having weight $60 \mathrm{~kg}$ receives hypoglycemia (hypo) episode at 10:30AM, and even after taking a sizeable snack at 10:00AM still had a low blood sugar at 11:00AM. He is also having somewhat high blood glucose levels in the evening. In this case we have to redistribute his current insulin regimen and/or food intake to reduce the risk of hypo during the mid-morning, while at the same time controlling his nocturnal blood sugars more tightly. The Table 1 illustrates the dietary plan of the above patient and its insulin doses are given in Table 2.

TABLE 1. DIETARY PLAN OF THE PATIENT DURING 24HRS

\begin{tabular}{|l|l|l|l|l|l|l|}
\hline Meals: & $\begin{array}{l}\text { Break } \\
\text { fast }\end{array}$ & $\begin{array}{l}\text { Snack } \\
\mathrm{s}-1\end{array}$ & $\begin{array}{l}\text { Lun } \\
\text { ch }\end{array}$ & $\begin{array}{l}\text { Snack } \\
\mathrm{s}-2\end{array}$ & $\begin{array}{l}\text { Dinne } \\
\mathrm{r}\end{array}$ & $\begin{array}{l}\text { Snack } \\
\mathrm{s}-3\end{array}$ \\
\hline $\begin{array}{l}\text { Time } \\
\text { (hh:m } \\
\text { m) }\end{array}$ & $07: 30$ & $10: 00$ & $\begin{array}{l}12: \\
00\end{array}$ & $16: 00$ & $19: 00$ & $22: 00$ \\
\hline $\begin{array}{l}\text { Carbo } \\
(\mathrm{gms})\end{array}$ & 20 & 20 & 40 & 20 & 40 & 20 \\
\hline
\end{tabular}

TABLE 2. REGIMEN OF INSULIN INDUCED EXOGENOUSLY

\begin{tabular}{|l|c|c|c|}
\hline Insulin Infusion (hh: mm) & $07: 30$ & $12: 00$ & $19: 00$ \\
\hline Insulin Dose Quantity (units) & 10 & 6 & 6 \\
\hline $\begin{array}{l}\text { Manipulated Insulin Dose Quantity } \\
\text { (units) }\end{array}$ & 3 & 6 & 6 \\
\hline
\end{tabular}

When the above case is simulated over ITM, the result shows that a hypo episode is encountered during the breakfast. This episode is due to high insulin induced exogenously during the breakfast. To achieve normoglycemia level the above insulin regimen is manipulated by lowering the units of insulin during the breakfast. As shown in Table 2 the insulin is reduced to 3 units from 10 units.

Now, by adopting the modified therapy, the patient blood glucose profile is simulated, the results shows that the blood glucose levels of the patient are now in normoglycemia range during the breakfast. The results are depicted in the Figure 6. 


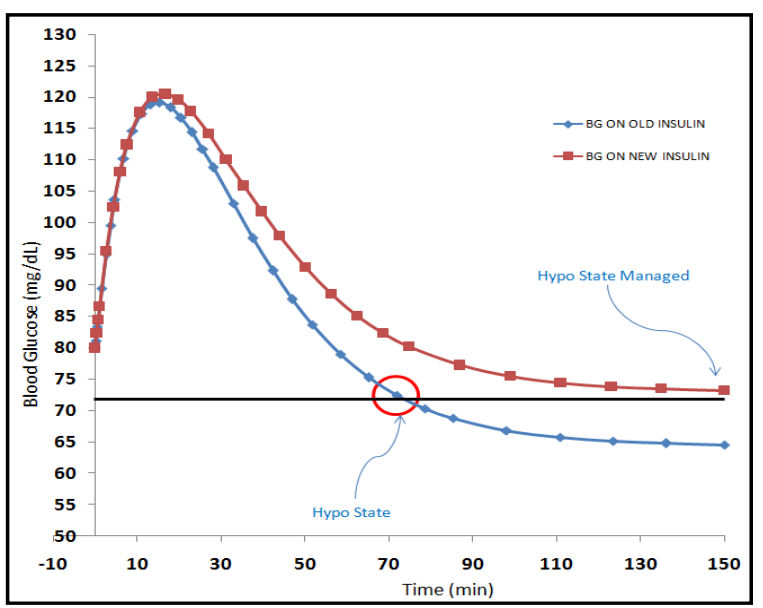

Fig 6: Variation in blood glucose values when insulin doses are manipulated. Decreasing the quantity of insulin increases the blood glucose level and hence reduces the chances of hypoglycemia state.

By simulating this case we have seen that ITM successfully models the blood glucose profile of the patient receiving hypoglycemia episodes. Similarly we can manage the other hypoglycemia episodes received during the $24 \mathrm{Hrs}$ blood glucose profile of the above case.

\section{Conclusion and Future Directions}

In this paper we discussed the design of ITM reasoning system. The design of ITM reasoning system uses Bergman minimal model [15], Fisher model [16] and Roy exercise model [17] as implementation for modeling the blood glucose concentration of patient having type 1 diabetes mellitus (T1DM). The ITM successfully captures the blood glucose profile of the patient. Finally the designed ITM was tested with the cases of existing AIDA simulator for checking its performance.

The future research related to enhancement of ITM can be carried out in following directions:

(i) The patient takes a mixed meal composed of carbohydrates, fats, proteins and other nutrients. These nutrients affect the blood glucose concentration. The ITM can be enhanced to include fats and proteins in the patient's dietary intake.

(ii) For providing the complete reasoning mechanism a knowledge base can also need to be maintained. (iii) The design of ITM was based on hybrid architecture for performing the tasks of temporal reasoning with limited use of temporal maintenance. Existing temporal maintenance systems (like TQuery, TSQL2 etc.) can also be integrates with the designed ITM for proper temporal data management.

\section{References}

[1] A. Rajak, K. Saxena. Achieving Realistic and Interactive Clinical Simulation using Case based Therasim's Therapy Engine Dynamically. In: Proceedings of the National Conference on Advanced Pattern Mining and Multimedia Computing, 2010, 624-628.

[2] A. Rajak, K. Saxena. Modeling Temporal Databases-A Survey. International Journal of Computer and Electronics Engineering, 2009, 1(1), 77-82.

[3] A. Rajak, K. Saxena. Managing Temporal Databases. In: Proceedings of the International Conference on Managing Next Generation Software Applications, 2008, 107-114.

[4] Y. Shahar, C. Combi. Temporal Reasoning and Temporal Data Maintenance in Medicine: Issues and Challenges. Computers in Biology and Medicine, 1997, 27(5), 353368.

[5] A. Rajak, K. Saxena. Research Issues Related to Temporal Mediators based on Clinical Domain. International Journal of Recent Trends in Engineering, 2009, 1(1), 535-540.

[6] A. Rajak, K. Saxena. Temporal Abstraction Database Mediators based on Clinical Guidelines. In: Proceedings of the IEEE International Advance Computing Conference, 2009, 2274-2279.

[7] A. Rajak, K. Saxena. Temporal Reasoning with Time Oriented Medical Database using Models based on Insulin-Glucose Metabolism. International Journal of Computer Science and Information Technology \& Security, 2011, 1(2), 66-70.

[8] A. Rajak, K. Saxena. Design of ITM Reasoning System based on Open-loop Insulin Delivery Method for T1DM. Accepted for publication.

[9] R. N. Bergman, G. M. Steil, A. Volund, S. E. Kahn. Reduced Sample Number for Calculation of Insulin Sensitivity and Glucose Effectiveness from the Minimal Model. Diabetes 42, 1993, 250-256.

[10] R. N. Bergman, G. Pacini. MINMOD: A Computer Program to Calculate Insulin Sensitivity and Pancreatic Responsivity from the Frequently Sampled Intravenous Glucose Tolerance Test. Computer Methods and Programs in Biomedicine 23, 1986, 113-122.

[11] R. N. Bergman, G. Toffolo, D. T. Finegood, C. R. Bowden, C. Cobelli. Quantitative Estimation of Beta Cell Sensitivity to Glucose in the Intact Organism-A Minimal Model of Insulin Kinetics in the Dog. Diabetes 29, 1980, 979-990.

[12] R. N. Bergman, L. S. Phillips, C. Cobelli. Physiological Evaluation of Factors Controlling Glucose Tolerance in Man. Journal of Clinical Investigation 68, 1981, 14561467.

[13] R. N. Bergman, M. F. Saad, R. L. Anderson, A. Laws, R. M. Watanabe, W. W. Kades, Y. D. I. Chen, R. E. Sands, D. Pei, P. J. Savage. A Comparison between the Minimal Model and the Glucose Clamp in the Assessment of Insulin Sensitivity across the Spectrum of Glucose Tolerance. Diabetes 43, 1994, 1114-21. 
[14] R. N. Bergman, Y. Z. Ider, C. R. Bowden, C. Cobelli. Quantitative Estimation of Insulin Sensitivity. American Journal of Physiology 236, 1979, E667-77.

[15] R. N. Bergman. Minimal Models for Glucose and Insulin Kinetics. http://www.civilized.com/mlabexamples/glucose.htmld/

[16] M. E. Fisher. A Semi-Closed Loop Algorithm for the Control of Blood Glucose Levels in Diabetes. IEEE Transactions on Biomedical Engineering 38, 1991, 57-61.

[17] A. Roy. Dynamic Modeling of Free Fatty Acid, Glucose, and Insulin during Rest and Exercise in Insulin Dependent Diabetes Mellitus Patients. Doctoral Dissertation, University of Pittsburgh, PhD, 2008.

[18] A. Rajak, K. Saxena. Modeling Clinical Database using Time Series based Temporal Mining. International Journal of Computer Theory and Engineering, 2010, 2(2), 185188.

[19] E. D. Lehmann, T. Deutsch. A Physiological Model of Glucose-Insulin Interaction in Type 1 Diabetes Mellitus. Journal of Biomedical Engineering, May 1992, 14(3), 235-242.

Akash Rajak is working as Associate Professor in Krishna Institute of Engineering and Technology, Ghaziabad. He completed M.C.A from U.T.D Campus, Dr. H. S. Gour University, Sagar (M.P) in 2002. He is pursuing his Ph.D in computer science from Barkatullah University, Bhopal in the field of temporal databases. He has more than 9 years of teaching and research experience. He is a life member of various professional societies like ACEEE, IACSIT, IAENG, CSTA etc. He is a reviewer of various international conferences, journals and books. He also served as program committee chair of various ACEEE conferences.

Kanak Saxena received her $\mathrm{Ph}$. D. in computer science from the Devi Ahilya University, Indore, India. She is professor in the Computer Applications Department at the Samrat Ashok Technological Institute affiliated to Rajiv Gandhi Technical University, Bhopal. Her current research focuses on database systems, parallel computing, data uncertainty and design and other interests include network security and performance and software engineering. She is the member of the editorial board of international journals and member of various international committees of international conferences. 